\title{
Large-scale simulations of quasi-brittle microcracking in realistic highly heterogeneous microstructures obtained from micro CT imaging
}

\author{
T.T. Nguyen ${ }^{a, b}, J$ Yvonnet ${ }^{a, *}$, M. Bornert $^{b}$, C. Chateau $^{\mathrm{b}}$ \\ F. Bilteryst ${ }^{c}$, E. Steib ${ }^{c}$ \\ ${ }^{a}$ Université Paris-Est, Laboratoire Modélisation et Simulation Multi Échelle \\ MSME UMR 8208 CNRS, 5 bd Descartes, 77454 Marne-la-Vallée, France. \\ ${ }^{\mathrm{b}}$ Université Paris-Est, Laboratoire Navier, CNRS UMR 8205, ENPC, IFSTTAR, \\ 6/8 avenue Blaise Pascal, 77455 Marne-la-Vallée, France. \\ ${ }^{\mathrm{c}}$ Université de Lorraine, Laboratoire d'Énergétique et de Mécanique Théorique et \\ Appliquée, LEMTA CNRS CNRS-UMR 7563, 27 rue d'Hellieule, 88100 Saint Dié \\ des Vosges, France.
}

\begin{abstract}
We present large-scale simulations of microcracking initiation and propagation using a continuum mechanics description in realistic, voxel-based microstructures of heterogeneous quasi-brittle materials obtained from CT imaging techniques. The phase field method is used to describe the evolution of the complex microcracks networks in both uniaxial tension and compression of a sub-volume of a lightweight concrete, where all pores and sand grains are explicitly described. A description of the meshing techniques for such complex voxel-based models is provided, and a convergence study of the tensile failure strength with respect to the sample size is carried out. Such large-scale simulations have high potential to be used either within recent concurrent multiscale methods or in approaches combining in-situ experiments with 3D imaging techniques and simulations for inverse identification of microstructural damage models.
\end{abstract}

Key words: Cracks, microstructures, phase field, quasi-brittle materials, image-based models of microstructures

* Correspondance to J. Yvonnet

Email address: julien.yvonnet@univ-paris-est.fr (J. Yvonnet ). 


\section{Introduction}

Predicting the strength of quasi-brittle materials such as concrete or civil engineering materials due to microcracking is a key issue in order to design concrete materials with extreme resistance properties. Such materials are inherently highly heterogeneous, multiscale and random by nature, which induces many challenges to predict their damage due to microcracking initiation and propagation. Extreme scale Direct Numerical Simulations (DNS) for cracking in materials have been proposed recently, and offer new opportunities to predict the macroscopic damage behavior by capturing all relevant microstructural physical mechanisms describing the evolution of the microstructure. Among them, the molecular dynamics (MD) can nowadays include billions of atoms $[1,2]$ and run over significant time ranges, and allows capturing detailed phenomena related to cracking such as crack branching, multiple cracks interacting, or ductile cracks with a fine description of microvoids coalescence. A review on MD for large-scale simulations, including brittle fracture, dynamic cracking and ductile failure can be found e.g. in [10]. Other particle-based methods like peridynamics [26] are alternatives to investigate complex cracking in fine models of brittle materials. Even though promising in view of the continuous growth of supercomputers capabilities, these simulations are still restricted so far to small spatial domains (lower than $1 \mu \mathrm{m}^{3}$ ), usually in the process zone of the crack.

On the other hand, continuum description of microcracking in heterogeneous materials allows simulating damage in larger samples with scales ranging from micrometers to millimeters with a higher number of inclusions/heterogeneities. Experimental imaging like X-Ray microtomography (see e.g. [12]) can nowadays be routinely used to provide a complete $3 \mathrm{D}$ realistic description of the morphology of heterogeneous materials and can serve as a basis to construct the numerical models employed in the simulations. Combined with 3D experimental imaging techniques, developing large-scale simulations of cracking also offers new routes to identify microstructural damage parameters by inverse approaches, from both macro and microstructural full fields, and then to use the microstructural models to conduct predictive simulations in the virtual materials paradigm. However, including all microscopic heterogeneities at the scale of a whole sample, i.e. all pores, gravels, sand grains, initial microcracks... present, for instance, in a concrete sample, is nowadays still out of reach. In $[17,16]$, Mosby and Matouš developed hierarchically parallel solvers based on multi-scale simulations on extreme scales in terms of both physical length scales and computed capabilities resources, and show the ability to efficiently compute the failure of heterogeneous samples from sub-micrometer to centimeter scales in damage mechanics problems of particle-reinforced adhesives. In [24], another multiscale framework using large-scale simulations at the microstructural level has been proposed to study cracking in a ultra-high 
strength steel.

Another possibility to overcome the above-mentioned computational limitations is to study a sub-volume of the whole sample which can be meshed and used for simulations in reasonable times, and to prescribe experimentally captured displacements obtained by 3D image-correlation techniques over the boundary of the sub-volume to conduct the simulations. In this context, the first quantitative comparison between 3D crack networks obtained by experiments and simulations at the continuum level in large-scales FEM models of lightweight concrete samples has been performed in [18].

The purpose of this letter is to present the current capability of large-scale simulations of microcracking in realistic microstructural models of lightweight concrete obtained from microtomography images. Such simulations can be either used within multiscale simulations such as in $[17,16,24]$, or in combined experiments/simulations sub-volume techniques as described in [18] to accurately describe the damage due to microscale cracking.

\section{Phase field modelling of quasi-brittle microcracking}

In the following, the basic concepts of the phase field method are briefly summarized. For more details and practical implementation aspects, the interested reader can refer to $[14,20]$. The phase field method is based on a regularized formulation of a sharp crack description. A regularized variational principle describes both the evolution of the mechanical problem and of an additional field $d$ describing the damage (called phase field). It is discretized by a finite element procedure and a staggered algorithm, chosen here due to its numerical efficiency. As compared to classical volume damage models, such regularized approach is directly connected to the theory of brittle crack propagation and removes mesh-sensitivity issues due to its natural nonlocal character.

In the phase field method, assuming small strains, the regularized form of the energy describing the cracked structure is expressed by:

$$
E(\mathbf{u}, d)=\int_{\Omega} W(\mathbf{u}, d) d \Omega+g_{c} \int_{\Omega} \gamma(d, \nabla d) d \Omega,
$$

where $W$ is the density of the elastic energy, depending on the displacements $\mathbf{u}(\mathbf{x})$ and on the phase field $d(\mathbf{x})$ describing the damage of the solid, $g_{c}$ is the fracture resistance and $\gamma(d, \nabla d)$ is the crack energy density, defined by $\gamma(d, \nabla d)=\frac{1}{2 \ell} d^{2}+\frac{l}{2} \nabla d \cdot \nabla d$ (see e.g. $\left.[14,20]\right)$.

Applying the principle of maximum dissipation and energy minimization [13] to (1) yields the set of coupled equations to be solved on the domain $\Omega$ as- 
sociated with the structure, with boundary $\partial \Omega$ and outward normal $\mathbf{n}$, to determine $d(\mathbf{x})$ and $\mathbf{u}(\mathbf{x}), \forall \mathbf{x} \in \Omega$ :

$$
\left\{\begin{array}{l}
2(1-d) \mathcal{H}-\frac{g_{c}}{\ell}\left\{d-\ell^{2} \Delta d\right\}=0 \text { in } \Omega \\
d(\mathbf{x})=1 \text { on } \Gamma \\
\nabla d(\mathbf{x}) \cdot \mathbf{n}=0 \text { on } \partial \Omega
\end{array}\right.
$$

and

$$
\left\{\begin{array}{l}
\nabla \cdot \boldsymbol{\sigma}(\mathbf{u}, d)=\mathbf{f} \text { in } \Omega \\
\mathbf{u}(\mathbf{x})=\overline{\mathbf{u}} \text { on } \partial \Omega_{u} \\
\boldsymbol{\sigma} \mathbf{n}=\overline{\mathbf{F}} \text { on } \partial \Omega_{F}
\end{array}\right.
$$

In (2), $\Gamma$ refers to the crack surface, $\ell$ is the regularization parameter. The history strain energy density function $\mathcal{H}(\mathbf{x}, t)$, where $t$ denotes time, is introduced to describe a dependence on history [14] and possible loading-unloading, and reads:

$$
\mathcal{H}(\mathbf{x}, t)=\max _{\tau \in[0, t]}\left\{\Psi^{+}(\mathbf{x}, \tau)\right\}
$$

In (4), $\Psi^{+}$is the tensile part of the elastic strain density function serving to model unilateral contact, and is defined as

$$
\Psi^{+}(\varepsilon)=\frac{\lambda}{2}\left(\langle\operatorname{Tr}(\varepsilon)\rangle_{+}\right)^{2}+\mu \operatorname{Tr}\left\{\left(\varepsilon^{+}\right)^{2}\right\}
$$

where $\varepsilon$ is the linearized strain tensor, $\langle x\rangle_{ \pm}=(x \pm|x|) / 2$ and $\varepsilon^{ \pm}$are compression and tensile parts of the strain tensor (see e.g. [14,20]). The choice of the numerical parameter $\ell$ has been discussed e.g. in $[5,9,21,19]$.

In (3), $\boldsymbol{\sigma}=\frac{\partial W}{\partial \varepsilon}$ is the second-order Cauchy stress tensor, $\mathbf{f}$ are body forces and $\overline{\mathbf{u}}$ and $\overline{\mathbf{F}}$ are prescribed displacements and forces on the corresponding boundaries $\partial \Omega_{u}$ and $\partial \Omega_{F}$, respectively. The symbols $\nabla($.$) and \nabla \cdot($.$) denote$ gradient and divergence operators, respectively. For the strain-density function (5), the constitutive law is expressed (see e.g. [14]) by:

$$
\boldsymbol{\sigma}=\left((1-d)^{2}+k\right)\left\{\lambda\langle\operatorname{Tr} \boldsymbol{\varepsilon}\rangle_{+} \mathbf{1}+2 \mu \boldsymbol{\varepsilon}^{+}\right\}+\lambda\langle\operatorname{Tr} \boldsymbol{\varepsilon}\rangle_{-} \mathbf{1}+2 \mu \boldsymbol{\varepsilon}^{-}
$$

where $k$ is a small positive numerical parameter serving to avoid loss of stability in case of fully damaged elements.

Eqs. (2)-(3) are solved by a standard FEM procedure in a staggered scheme at each time step (load increment). 


\section{Construction of the numerical model from experimental 3D im- ages}

In the present work, the above equations are solved within realistic geometrical models of microstructures directly obtained from X-Ray micro computated tomography (XR- $\mu \mathrm{CT}$ ) of lightweight concrete (see details of the experimental procedure in [18]). For this purpose, finite elements meshes must be constructed from the segmented images, which are obtained by applying filters or morphological operators on the grey-level initial images. Details about such techniques can be found e.g. in [3]. Then, two techniques can be employed to construct the FEM meshes. The first one consists in converting each voxel of the segmented image into a cubic domain, which can be associated with one or several 8-node elements or which can be decomposed into tetrahedra. Then, the properties defined in each phase from the segmented image are mapped onto the cubic domains. The advantages of this strategy are its simplicity and the possibility to use parallel solving procedures related to regular meshes in the numerical simulations. The main drawback comes from the obtained large meshes. Adaptive mesh refinement is possible in such regular meshes through e.g. octree meshes [8]. Accuracy of voxel-based models has been studied e.g. in $[23]$.

The second strategy is to use a meshing software able to work on initial data in the form of 3D voxel-based segmented image, and which is able to reconstruct

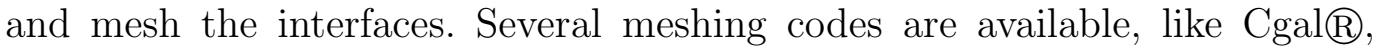
Tetgen®, Iso2mesh®, AVIZOß,... In the present work, the AVIZOß software has been used. An illustration of the application of such a technique to a segmented 3D image of lightweight concrete is depicted in Fig. 1. The main advantage of this technique is that a lower number of mesh elements can be obtained as compared to regular meshes, by exploiting the reconstructed interfaces and by refining regions of interest. The drawback is that these meshing codes lack robustness for very complex geometries or for domaines containing too many separated phases. A comparative study of both techniques can be found e.g. in [25]. In this work, we present examples using both approaches.

First, several meshes are constructed by extracting subdomains in the voxelized image of the undamaged microstructure. To guarantee comparable mesh accuracy between the different simulations, we use here regular meshes by directly projecting the voxel material properties on a regular mesh of 8node finite elements. Then, several samples with an increasing number of voxels are defined, denoted by 0.5M_STRUCT, 5M_STRUCT, 9M_STRUCT, 17M_STRUCT, 30M_STRUCT (see Fig. 2). The samples involve respectively $0.576 \times 10^{6}, 5.376 \times 10^{6}, 9 \times 10^{6}, 17.712 \times 10^{6}$ and $29.7 \times 10^{6}$ voxels, which corresponds to the same number of 3D 8-node finite elements. The average element size is $20 \mu \mathrm{m}$. 


\begin{tabular}{lllll}
\hline Mesh & Nb. elements & $a[\mathrm{~mm}]$ & $b[\mathrm{~mm}]$ & $c[\mathrm{~mm}]$ \\
\hline 0.5M_STRUCT & 576000 & 1.40 & 1.40 & 1.75 \\
5M_STRUCT & 5376000 & 2.80 & 2.80 & 4.90 \\
9M_STRUCT & 9000000 & 3.50 & 3.50 & 5.25 \\
17M_STRUCT & 17712000 & 4.20 & 4.20 & 7.18 \\
30M_STRUCT & 29700000 & 5.25 & 5.25 & 7.70 \\
17M_UNSTRUCT & 16952513 & 5.25 & 6.00 & 7.80 \\
\hline
\end{tabular}

Table 1

Characteristics of the different meshes and corresponding domain dimensions, where $a, b$ and $c$ denote dimensions in $x, y$ and $z$ directions, respectively.

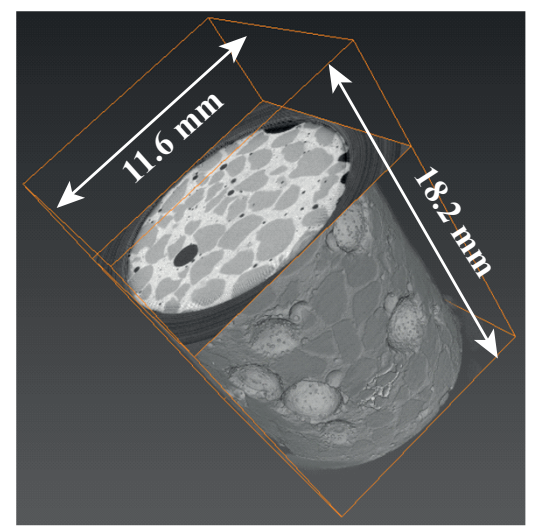

(a)

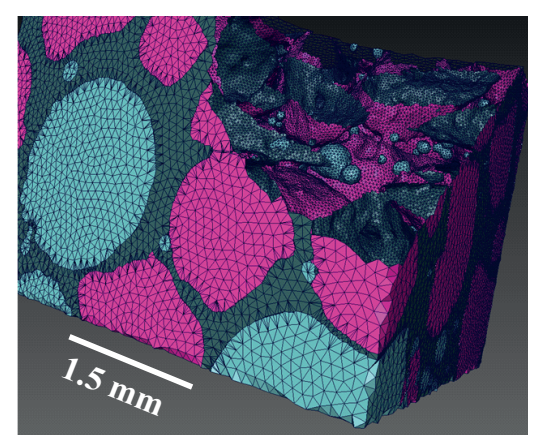

(c)

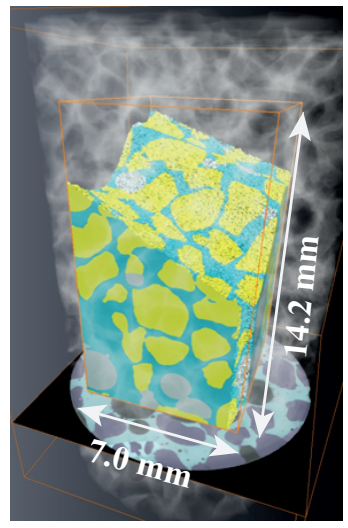

(b)

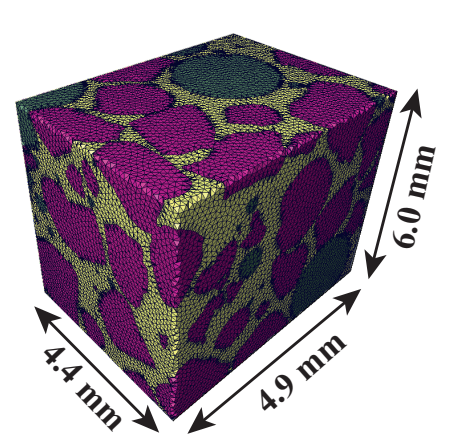

(d)

Fig. 1. (a) Full CT image of a lighweight concrete; (b)-(c)-(d) views of the unstructured mesh 17M_UNSTRUCT constructed from the CT image.

In addition, an unstructured mesh is obtained using the first technique described above. This mesh, denoted as 17M_UNSTRUCT, is composed of $17.10^{6}$ tetrahedral elements. (The characteristic length of elements is $25 \mu \mathrm{m}$ in the region of expected crack path (interface of inclusions and pores) and $60 \mu \mathrm{m}$ inside inclusions and pores). The characteristics of the different meshes and corresponding domain dimensions are described in Table 1. 


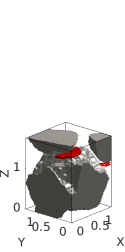

(a)

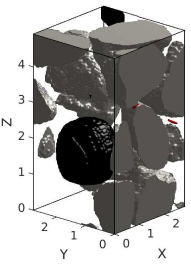

(b)

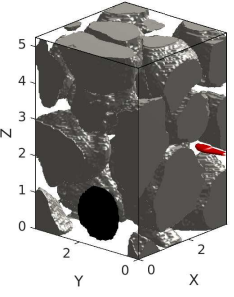

(c)

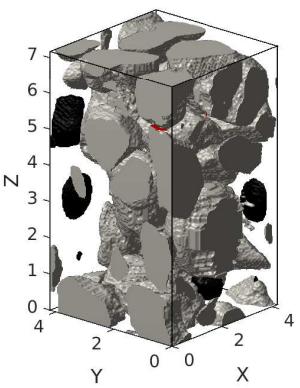

(d)

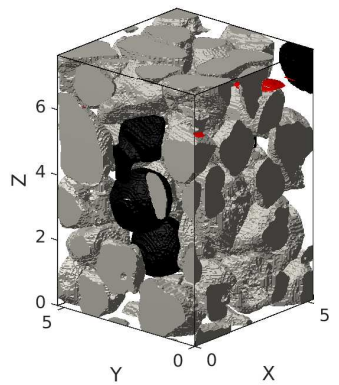

(e)

Fig. 2. Several sub-volumes cut in the whole voxel-based microstructural model: (a) 0.5M_STRUCT; (b) 5M_STRUCT; (c) 9M_STRUCT; (d) 17M_STRUCT and (e) 30M_STRUCT. Units on the axes are in $\mathrm{mm}$.

\section{Results}

In this section, the mechanical responses of the different samples defined from the above voxel models of a lightweight concrete material are computed. The considered material is composed of a cement matrix, sand inclusions and EPS (expanded polystyrene) beads assimilated to the pores (details about the preparation of the samples can be found in [18]). A picture of a real sample is depicted in Fig. 3 (a). The material parameters of inclusions and matrix are the same as those used in [18], and are, respectively: $E_{i}=60 \mathrm{GPa}$, $\nu_{i}=0.3, E_{m}=18 \mathrm{GPa}$, and $\nu_{m}=0.2$. In the case of a full mesh of the microstructure from the voxel image, the pores do not contain any element. In the case of regular meshes matching the voxels, the pores are modeled as phases with very compliant properties taken as $E_{p}=10^{-6} \mathrm{GPa}, \nu_{p}=0$. The fracture energy $g_{c}=59.3 \mathrm{~N} / \mathrm{m}$ is assumed to be identical in the different phases. The regularization parameter is chosen as $\ell=30 \mu \mathrm{m}$. In the simulation, the following boundary conditions have been used to prescribe uni-axial compression and tension: on the lower end $\left(z=z_{\min }\right)$, the $z$-displacements are blocked, while the $x$-displacements and $y$-displacements are free. On the upper end, the $x$-displacements and $y$-displacements are free, while the $z$-displacements are prescribed, with an increasing value of $\Delta \bar{U}$ during the simulation: $\Delta \bar{U}=10^{-5} \mathrm{~mm}$ for the tensile case and $\Delta \bar{U}=-2.10^{-5} \mathrm{~mm}$ for the compression case (see Fig. 3 (b)). The geometry of the phases is the one depicted in Fig. 3 (c).

We first conduct a uniaxial tension test on a large sub-volume of size $a=5.25$ $\mathrm{mm}, b=6 \mathrm{~mm}, c=7.8 \mathrm{~mm}$, meshed with an unstructured mesh obtained with Avizoß, containing $17.10^{6}$ tetrahedral elements and $9.10^{6}$ degrees of freedom. Fig. 4 (a) provides a representation of the rather complex computed crack network (i.e. elements with $d=1$ ) developping throughout the sample, for various loading stages. We recall that within the phase field context, the different microcracks propagate and merge without any need of prior information 


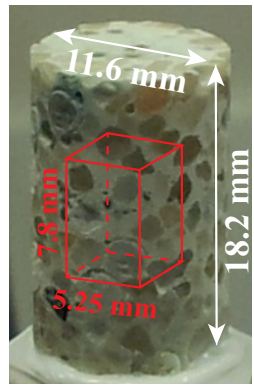

(a)

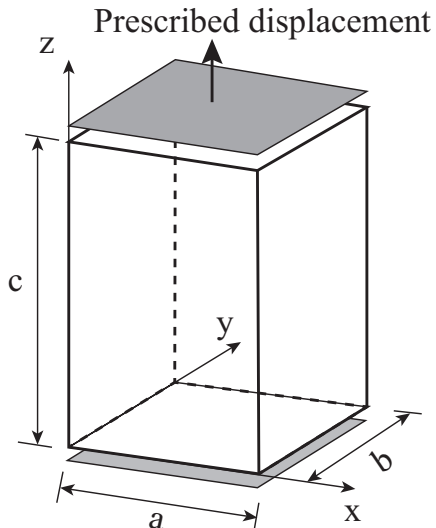

(b)

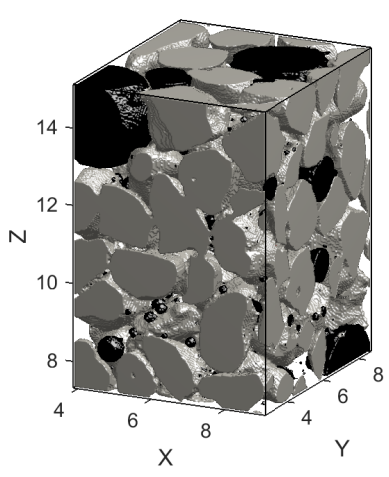

(c)

Fig. 3. Microtomography image-based concrete sample in 3D: (a) real sample, (b) boundary conditions for uniaxial or compression tests (see a detailed description in the text) and (c) geometry of the sub-volume extracted from the sample.

about their location.

In Fig. 4 (b), the morphology of the microcracks network induced by compression in the same sample 17M_UNSTRUCT is depicted. We again observe very complex micro cracking patterns, but the morphology is different from the tensile test: in this case, the microcracks are roughly aligned with the direction of the load, while in the tensile test they propagate in direction perpendicular to the load. This is in agreement with other observations in more simple heterogeneous quasi-brittle samples [19].

First, we study the convergence of the response of one parallelepipedic domain cut in the whole sample with respect to the mesh size. For this purpose, the mesh 9M_STRUCT has been used, and the different mesh sizes have been obtained by coarsening the grid corresponding to the 3D segmented image $\left(h_{e}=0.01 \mathrm{~mm}\right)$. For coarse meshes, the material properties of the elements containing different initial phases are defined as the property of the highest fraction of one phase in the element. Results are presented in Figs. 5 and 6. We can note that the values obtained with the initial mesh correspond to roughly converged values of the maximum tensile strength.

Next, we study the statistical representativity of the samples. For this purpose, we have cut 8 domains of the same size than 9M_STRUC taken at different locations in the whole sample. Results are presented in Fig. 7 where the mean and standard deviation associated with the 8 realizations are depicted. We can note that the error related to statistical representativity (by performing a simulation on one single realization), is around $11 \%$ for this size of domain.

We now study the convergence of the stress response with respect to the size of a parallelepipedic domain cut in the sample, for a fixed mesh size $\left(h_{e}=0.01\right.$ 

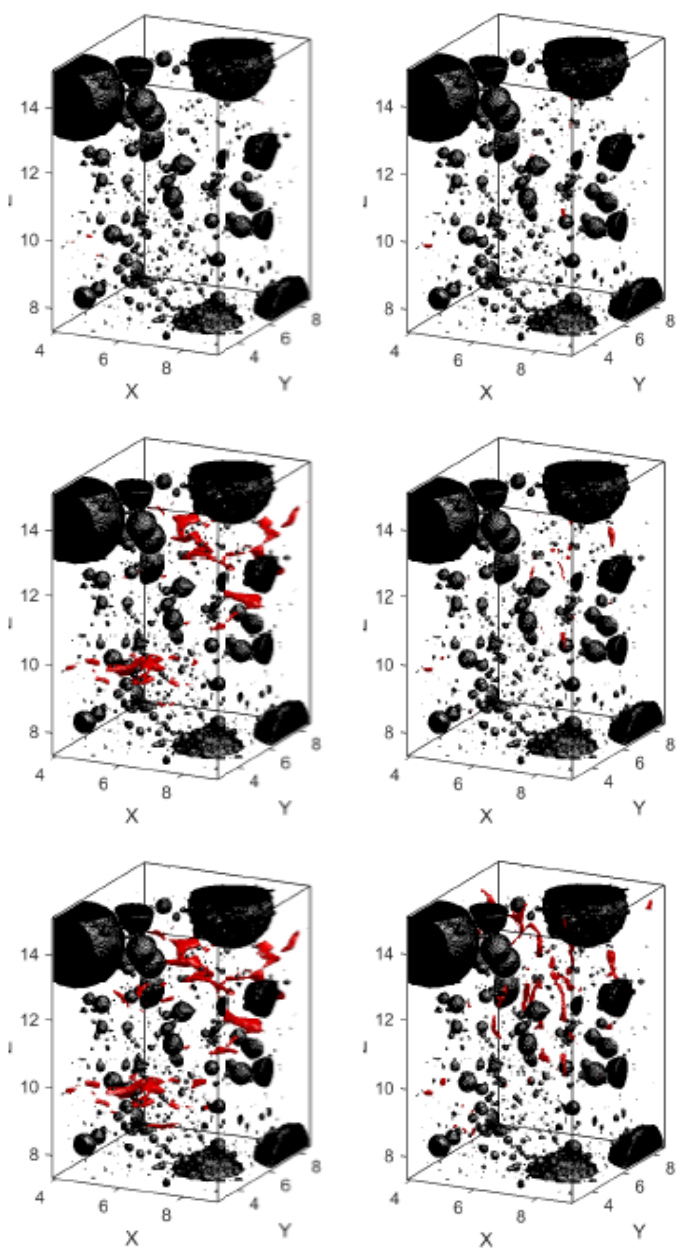

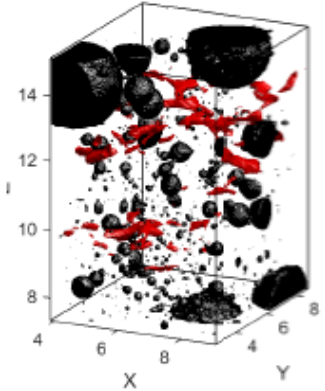

(a)

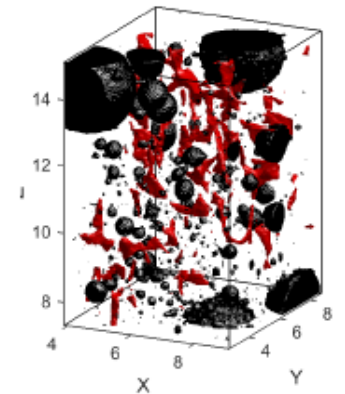

(b)

Fig. 4. Numerical prediction of crack network in a model based on a microtomography image of a real lightweight concrete sample. Black, and red colors refer to pores and cracks, respectively. Sand grains are not depicted for the sake of clarity. Red zones correspond to $d=1$; (a) Crack network propagation under tension for prescribed displacements (top to bottom): $\mathrm{u}=1.5 \mu \mathrm{m}\left(\bar{\varepsilon}_{z z}=1.925 \times 10^{-4}\right) ; \mathrm{u}=1.6 \mu \mathrm{m}$ $\left(\bar{\varepsilon}_{z z}=2.053 \times 10^{-4}\right) ; \mathrm{u}=1.65 \mu \mathrm{m}\left(\bar{\varepsilon}_{z z}=2.12 \times 10^{-4}\right) ; \mathrm{u}=1.7 \mu \mathrm{m}\left(\bar{\varepsilon}_{z z}=2.18 \times 10^{-4}\right)$; (b) Crack network propagation in compression for prescribed displacements (top to bottom): $\mathrm{u}=6 \mu \mathrm{m}\left(\bar{\varepsilon}_{z z}=-7.7 \times 10^{-4}\right) ; \mathrm{u}=6.3 \mu \mathrm{m}\left(\bar{\varepsilon}_{z z}=-8.1 \times 10^{-4}\right) ; \mathrm{u}=6.6$ $\mu \mathrm{m}\left(\bar{\varepsilon}_{z z}=-8.5 \times 10^{-4}\right) ; \mathrm{u}=6.9 \mu \mathrm{m}\left(\bar{\varepsilon}_{z z}=-8.9 \times 10^{-4}\right)$. Units on the axes are in $\mathrm{mm}$. 


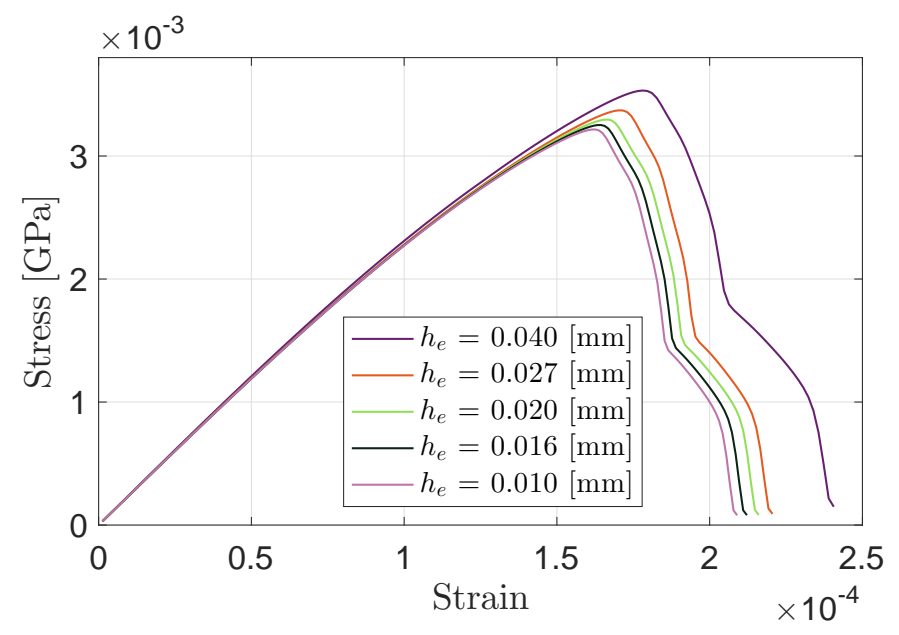

Fig. 5. Stress - strain curves response of the sample 9M_STRUCT for different mesh refinements.

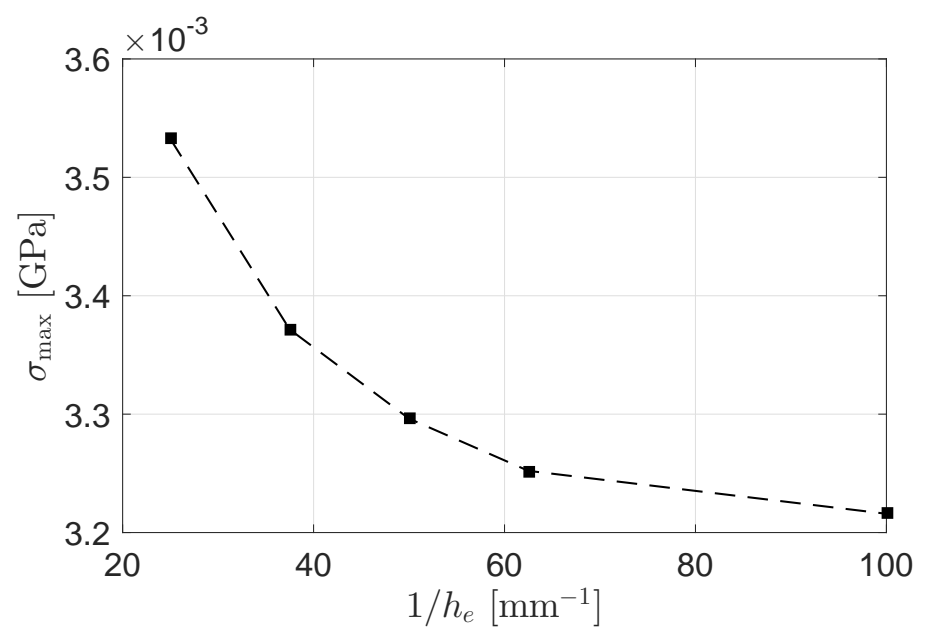

Fig. 6. Convergence of the maximum tensile stress for the sample 9M_STRUC with respect to the mesh size.

$\mathrm{mm}$ ). For this purpose, the 0.5M_STRUCT, 5M_STRUCT, 9M_STRUCT, 17M_STRUCT and 30M_STRUCT meshes whose size are increasing have been used. Due to the high computational costs involved and in view of the reasonable errors related to statistical representativity and mesh refinement, only one mesh size and one realization for each size of sample have been used.

In Fig. 8 the stress-strain curves of the different samples are presented in both compression and tension regimes. To analyze more clearly the response in tension, a zoom of Fig. 8 in the tensile regime is depicted in Fig. 9. It is observed that while the response of the 0.5M_STRUCT sample strongly differs from the other samples responses, which is expected as the size of 0.5M_STRUCT is very small and does not contain pores, the various other response are close to each other and seem to converge towards the response of the large 30M_STRUCT, as the size of the computational cell increases. 


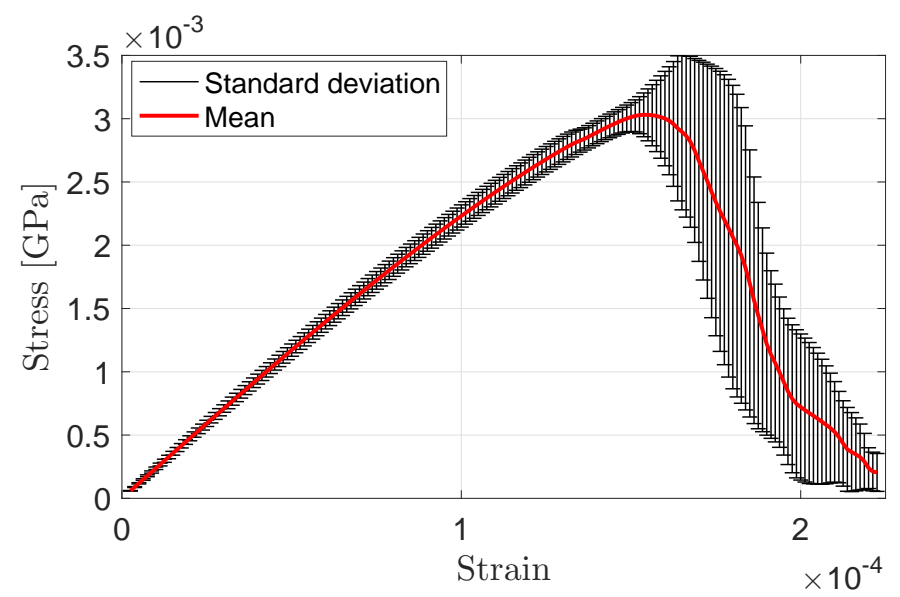

Fig. 7. Stress - strain curves response for different samples of same size taken in different locations of the whole sample.

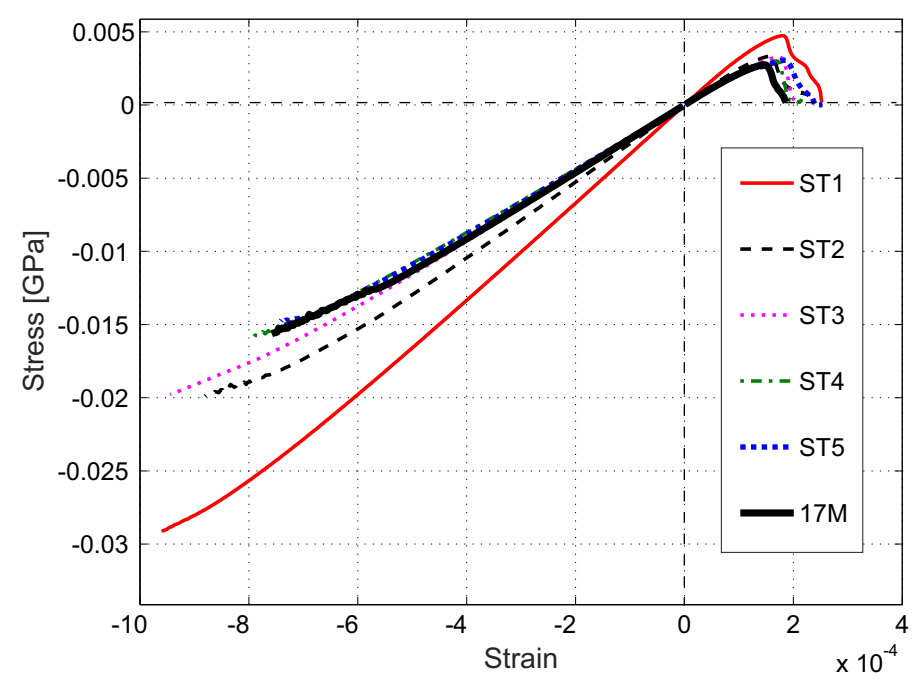

Fig. 8. Stress - strain curves for the different 3D microstructures under compression and tensile test.

This is true both in terms of stress-strain relation, and in particular for the initial linear modulus, and for failure stress. Of the latter, a clear dissymmetry between tension and compression is observed, consistently with experimental observations on cement based materials. Note that in tension the failure stress can be easily identified since it coincides with a maximum stress. However, in compression (see Fig. 4 (b)), due to the fact that the cracks propagate in the direction of the load, no clear failure point can be identified, as even when the sample is fully broken, it continues to have a resistance. Note that in this case the calculations are stopped after a pre-defined given number of time steps.

To better appreciate the convergence of the response, the values of the maximum tensile strength are reported in Fig. 10 for the different samples, and 


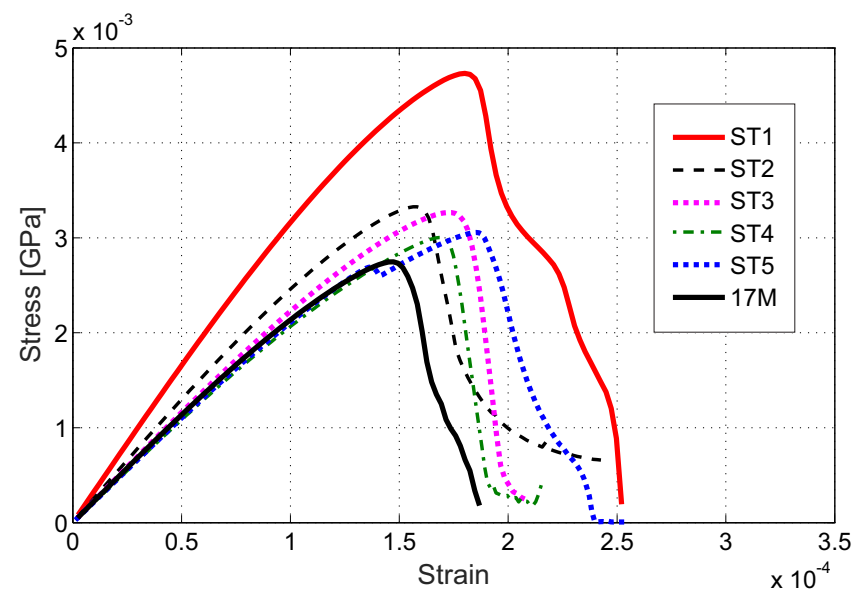

Fig. 9. Stress - strain curves for the different 3D microstructures (uniaxial tension).

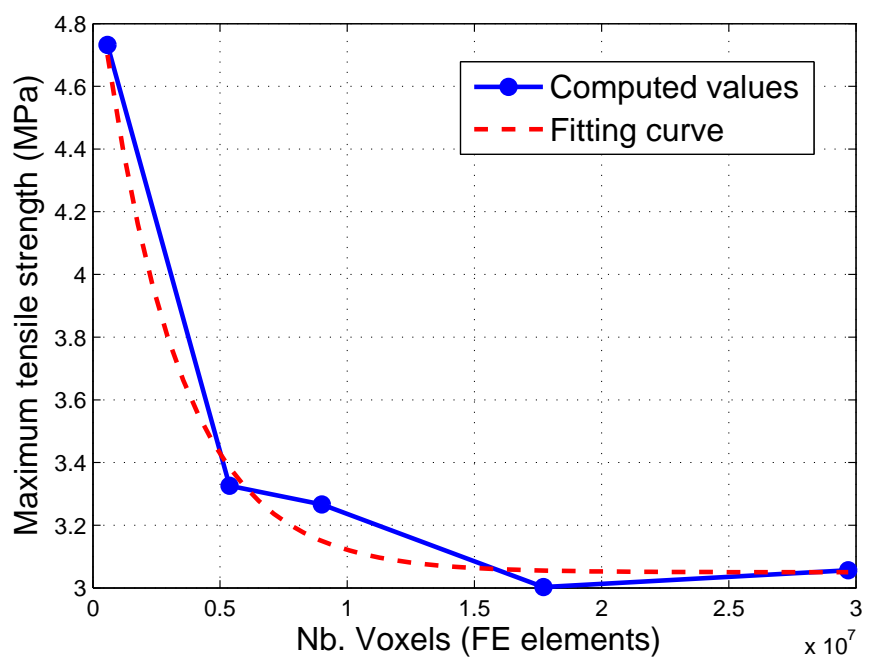

Fig. 10. Convergence of the maximum tensile strength (in $\mathrm{MPa}$ ) of the material with respect to the size of the model (nb. of elements)

plotted versus the number of voxels (elements) included in the model. We can appreciate the convergence of the results with respect to the sample size. Using a fitting curve $R=A+B \exp (-C . N)$ with $R$ the maximum tensile strength, $N$ the number of voxels (elements) and $A, B, C$ fitting parameters, we can extrapolate the converged value as $R=3.05 \mathrm{MPa}$. This value is in good agreement with available experimental values $[11,7,6]$. We note that the maximum stress of the 17M_UNSTRUCT sample appears however slightly lower than the others (about 2.8 MPa to be compared to 3.05 MPa). This might be related to the fact that the microstructures are slightly different and the meshes are constructed in a different manner (regular mesh against reconstructed-surfaces-based mesh).

Finally, a summary of the computational times for the different examples is reported in Table 2 . The presented results have been obtained with a home-made 


\begin{tabular}{llll}
\hline Problem & $\begin{array}{l}\text { Nb. time steps } \\
\text { (increments) }\end{array}$ & $\begin{array}{l}\text { CPU time for } \\
\text { one time step (s) }\end{array}$ & $\begin{array}{l}\text { Total simulation } \\
\text { time }(\mathrm{h})\end{array}$ \\
\hline 0.5M_STRUCT Tensile test & 90 & 70 & 1.75 \\
0.5M_STRUCT Compression & 225 & 70 & 4.5 \\
5M_STRUCT Tensile test & 135 & 860 & 32.25 \\
5M_STRUCT Compression & 245 & 860 & 58.5 \\
9M_STRUCT Tensile test & 125 & 1900 & 66 \\
9M_STRUCT Compression & 250 & 1900 & 132 \\
17M_STRUCT Tensile test & 115 & 3800 & 121.5 \\
17M_STRUCT Compression & 250 & 3800 & 163.5 \\
30M_STRUCT Tensile test & 140 & 9200 & 357.5 \\
30M_STRUCT Compression & 260 & 9200 & 664.5 \\
17M_UNSTRUCT Tensile test & 85 & 4100 & 96.5 \\
17M_UNSTRUCT Compression & 230 & 4100 & 262 \\
\hline Tam
\end{tabular}

Table 2

Size of computational cells, number and size of elements and computational times.

FE implementation of the numerical method within the Matlab®software, running on a workstation with 256 Go RAM and a single 8-core CPU cloked at $3 \mathrm{GHz}$. It shows that the presented simulations can be conducted on classical machines, but that a very large space for improvement of computational times is possible, by using massively parallel computers, such as e.g. in $[15,17]$.

\section{Conclusion}

In this letter, we have conducted large microcracking simulations in realistic voxel-based models of realistic microstructures of lightweight concrete. The simulations employ the continuum mechanics-phase field method, which allows simulating much larger domains than e.g. molecular dynamics. The simulations involve sub-volumes up to $7.7 \times 5.25 \times 5.25=212 \mathrm{~mm}^{3}$ with about $10 \mu \mathrm{m}^{3}$ resolution, and the largest simulations involve around 30 million elements. Such large-scale simulations have high potential to predict e.g. the fracture strength of sample by studying convergence of the response with respect to the sample size and can be used in accurate multiscale methods where fine fracturing process is required in some part of the domain, or in recent combined experimental 3D imaging and numerical simulations to identify microstructural damage models. Such a tool might also be very usefull to 
explore the links between local and global fracture properties in heterogeneous materials, which is still a widely open question.

\section{6 acknowledgements}

This work has benefited from a French government grant managed by ANR within the frame of the national program Investments for the Future ANR-11LABX-022-01. The financial support of J. Yvonnet from IUF (Institut Universitaire de France) is gratefully acknowledged.

\section{References}

[1] F. Abraham. Very large scale simulations of materials failure. Philos. T. R. Soc. A, 360(1792):367-382, 2002.

[2] F.F. Abraham, R. Walkup, H. Gao, M. Duchaineau, T. Diaz De La Rubia, and M. Seager. Simulating materials failure by using up to one billion atoms and the world's fastest computer: Brittle fracture. P. Natl. Acad. Sci. USA, 99(9):5777-5782, 2002.

[3] M.D. Abràmoff, P.J. Magalhães, and S.J. Ram. Image processing with ImageJ. Biophotonics international, 11(7):36-42, 2004.

[4] M.F. Adams, H. Bayraktar, T.M. Keaveny, and P. Papadopoulos. Ultrascalable implicit finite element analyses in solid mechanics with over a half a billion degrees of freedom. In Proceedings of the 2004 ACM/IEEE conference on Supercomputing, page 34. IEEE Computer Society, 2004.

[5] H. Amor, J.-J. Marigo, and C. Maurini. Regularized formulation of the variational brittle fracture with unilateral contact: Numerical experiments. $J$. Mech. Phys. Solids, 57(8):1209-1229, 2009.

[6] D.S. Babu, K.G. Babu, and W. Tiong-Huan. Effect of polystyrene aggregate size on strength and moisture migration characteristics of lightweight concrete. Cement Concrete Comp., 28(6):520-527, 2006.

[7] S.D. Babu, K.G. Babu, and T.H. Wee. Properties of lightweight expanded polystyrene aggregate concretes containing fly ash. Cement Concrete Res., 35(6):1218-1223, 2005.

[8] J. Bielak and O. Ghattas E.J. Kim. Parallel octree-based finite element method for large-scale earthquake ground motion simulation. CCMES-Comp. Model. Eng., 10(2):99, 2005. 
[9] M.J. Borden, C.V. Verhoosel, M.A. Scott, T.J.R. Hughes, and C.M. Landis. A phase-field description of dynamic brittle fracture. Comput. Meth. Appl. Mech. Eng., 217:77-95, 2012.

[10] M.J. Buehler and H. Gao. Ultra-large scale simulations of dynamic materials failure. Handbook of Theoretical and Computational Nanotechnology, 10:1-41, 2005 .

[11] B. Chen and C. Fang. Mechanical properties of eps lightweight concrete. Proceedings of the Institution of Civil Engineers-Construction Materials, 164(4):173-180, 2011.

[12] V. Cnudde and M.N. Boone. High-resolution x-ray computed tomography in geosciences: a review of the current technology and applications. Earth Sc. Rev., 123:1-17, 2013.

[13] G.A. Francfort and J.J. Marigo. Revisiting brittle fracture as an energy minimization problem. J. Mech. Phys. Solids, 46(8):1319-1342, 1998.

[14] C. Miehe, M. Hofacker, and F. Welschinger. A phase field model for rateindependent crack propagation: Robust algorithmic implementation based on operator splits. Comput. Meth. Appl. Mech. Eng., 199:2776-2778, 2010.

[15] M. Mosby and K. Matouš. Hierarchically parallel coupled finite strain multiscale solver for modeling heterogeneous layers. Int. J. Numer. Meth. Engng, 102(34):748-765, 2014.

[16] M. Mosby and K. Matouš. On mechanics and material length scales of failure in heterogeneous interfaces using a finite strain high performance solver. Mode. Simul. Mater. Sc., 23(8):085014, 2015.

[17] M. Mosby and K. Matouš. Computational homogenization at extreme scales. Extr. Mat. Lett., 6:68-74, 2016.

[18] T.T. Nguyen, J. Yvonnet, M. Bornert, and C. Chateau. Direct comparisons of $3 \mathrm{D}$ crack networks propagation in cementitious materials between phase field numerical modeling and in-situ microtomography experimental images. J. Mech. Phys. Solids, 95:320-350, 2016.

[19] T.T. Nguyen, J. Yvonnet, M. Bornert, C. Chateau, K. Sab, R. Romani, and R. Le Roy. On the choice of parameters in the phase field method for simulating crack initiation with experimental validation. Int. J. Fract., 197(2):213-226, 2016 .

[20] T.T. Nguyen, J. Yvonnet, Q.-Z. Zhu, M. Bornert, and C. Chateau. A phase field method to simulate crack nucleation and propagation in strongly heterogeneous materials from direct imaging of their microstructure. Eng. Frac. Mech., 139:1839, 2015.

[21] K. Pham, J.-J. Marigo, and C. Maurini. The issues of the uniqueness and the stability of the homogeneous response in uniaxial tests with gradient damage models. J. Mech. Phys. Solids, (6), 2011. 
[22] M. Rasquin, C. Smith, K. Chitale, E.S. Seol, B. Matthews, J. Martin, O. Sahni, R. Loy, and M.S. Shephard K.E. Jansen. Scalable fully implicit finite element flow solver with application to high-fidelity flow control simulations on a realistic wind design. Comput. Sci. Eng., 16(6):13-21, 2014.

[23] R.E. Guldberg S.H., Hollister, and G.T. Charras. The accuracy of digital imagebased finite element models. J. Biomed. Eng., 120:289-295, 1998.

[24] R. Tian and C. Wang. Large-scale simulation of ductile fracture process of microstructured materials. Progresses on Nuclear Sciences and Technologies, 2:24-29, 2011.

[25] M. Viceconti, L. Bellingeri, L. Cristofolini, and A. Toni. A comparative study on different methods of automatic mesh generation of human femurs. Medical engineering \&s physics, 20(1):1-10, 1998.

[26] X. Zhou, Y. Wang, and Q. Qian. Numerical simulation of crack curving and branching in brittle materials under dynamic loads using the extended nonordinary state-based peridynamics. Eur. J. Mech. A-Solid, 60:277-299, 2016. 